\title{
Genotype and Allele Frequencies of CYP2C9, VKORC1, CYP2C19 and PON1 in Chinese Han Population Detected in our Hospital
}

\author{
Keguang Chen, Rui Zhang, Nan Guo and Rui-chen Guo* \\ Institute of Clinical Pharmacology, Qilu Hospital of Shandong University, China
}

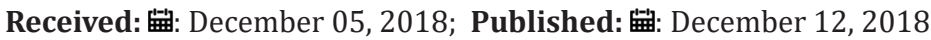

*Corresponding author: Rui-chen Guo, Institute of Clinical Pharmacology, Qilu Hospital of Shandong University, Jinan, China

\section{Opinion}

Warfarin and clopidogrel are commonly used anticoagulant and antiplatelet drugs, and their response and/or toxicity were significant influenced by genetic polymorphism of drug metabolizing enzymes transporters and drug targets. Different human populations can differ significantly in genetic allelic frequencies of these enzymes. This paper focuses on genotype and allele frequencies of genes which affect the pharmacokinetic and pharmacodynamic of warfarin or clopidogrel in Han Chinese population detected in our hospital, to provide guidance for the application of pharmacogenomics and clinical pharmacology.

\section{Materials and Methods}

All subjects, Han population and nonrelative, were inpatients or outpatients taking warfarin and/or clopidogrel at Qilu Hosiptal of Shandong University. Genetic polymorphisms of related enzymes were detected by fluorescence in situ hybridization (FISH).

\section{Results and Discussion}

\section{CYP2C9 and VKORC1}

Polymorphisms of cytochrome P450 2C9 (CYP2C9) and vitamin $\mathrm{K}$ epoxide reductase complex subunit 1 (VKORC1) genes are closely related to individualized treatment with warfarin [1]. Since CYP2C9*2 mutation is almost undetectable in Asian populations [2], we tested CYP2C9*3 (1075A >C, rs1057910) and VKORC1 (-1639G>A, rs9923231) for warfarin therapy. Results were shown in Tables 1 and 2. There was no significant difference in CYP2C9*3 gene mutation between male and female patients. Mutation frequency of A allele in VKORC1 $(-1639 \mathrm{G}>\mathrm{A})$ is higher in males than in females.

Table 1: Allele and genotype frequencies of CYP2C9*3 (1075A>C).

\begin{tabular}{|c|c|c|c|c|c|}
\hline \multirow{2}{*}{} & \multicolumn{3}{|c|}{ Genotype frequencies } & \multicolumn{2}{c|}{ Allele frequencies } \\
\cline { 2 - 6 } & AA & AC & CC & A & C \\
\hline Males $(n=104)$ & $90.39 \%(n=94)$ & $7.69 \%(n=8)$ & $1.92 \%(n=2)$ & $94.23 \%$ & $5.77 \%$ \\
\hline Females $(n=59)$ & $91.53 \%(n=54)$ & $6.78 \%(n=4)$ & $1.69 \%(n=1)$ & $94.91 \%$ & $5.08 \%$ \\
\hline Total patients $(n=163)$ & $90.80 \%(n=148)$ & $7.36 \%(n=12)$ & $1.84 \%(n=3)$ & $94.48 \%$ & $5.52 \%$ \\
\hline
\end{tabular}

Table 2: Allele and genotype frequencies of VKORC1 (-1639G>A).

\begin{tabular}{|c|c|c|c|c|c|}
\hline \multirow{2}{*}{} & \multicolumn{3}{|c|}{ Genotype frequencies } & \multicolumn{2}{c|}{ Allele frequencies } \\
\cline { 2 - 6 } & GG & GA & AA & G & A \\
\hline Males $(n=104)$ & $2.89 \%(n=3)$ & $14.42 \%(n=15)$ & $82.69 \%(n=86)$ & $10.10 \%$ & $89.90 \%$ \\
\hline Females $(n=59)$ & $3.39 \%(n=2)$ & $20.34 \%(n=12)$ & $76.27 \%(n=45)$ & $13.56 \%$ & $86.44 \%$ \\
\hline Total patients $(n=163)$ & $3.07 \%(n=5)$ & $16.56 \%(n=27)$ & $80.37 \%(n=131)$ & $11.35 \%$ & $86.65 \%$ \\
\hline
\end{tabular}




\section{YP2C19 and PON1}

CYP2C19 [3,4] and paraoxonase 1(PON1 A>G, rs662) [5] gene detection are mainly used for clinical medicine of clopidogrel. CYP2C19*2(681G>A, rs4244285) and CYP2C19*3(636G>A rs4986893) are the most common loss-of-function mutations [3], while CYP$2 \mathrm{C} 19 * 17(-806 \mathrm{C}>\mathrm{T}$, rs12248560) was associated with increased enzyme activity [5]. Allele and genotype frequencies of CYP2C19 results were shown in Tables 3-5. Metabolic phenotype frequencies of CYP2C19 were shown in Table 6. Allele and genotype frequencies of PON1 results were shown in Table 7. These results showed that mutation frequency of A allele in CYP2C19*3(636G>A) is higher in males than in females. Metabolic phenotype of CYP2C19*2/*2 is significantly higher in females than in males. And mutation frequency of A allele in $\mathrm{PON} 1(\mathrm{~A}>\mathrm{G})$ is higher in females than in males.

Table 3: Allele and genotype frequencies of CYP2C19*2(681G>A).

\begin{tabular}{|c|c|c|c|c|c|}
\hline \multirow{2}{*}{} & \multicolumn{3}{|c|}{ Genotype frequencies } & \multicolumn{2}{c|}{ Allele frequencies } \\
\cline { 2 - 6 } & GG & GA & AA & G & $30.61 \%$ \\
\hline Males $(\mathrm{n}=196)$ & $45.92 \%(\mathrm{n}=90)$ & $46.94 \%(\mathrm{n}=92)$ & $7.14 \%(\mathrm{n}=14)$ & $69.39 \%$ & $37.32 \%$ \\
\hline Females $(\mathrm{n}=71)$ & $39.44 \%(\mathrm{n}=28)$ & $46.48 \%(\mathrm{n}=33)$ & $14.08 \%(\mathrm{n}=10)$ & $62.68 \%$ & $32.40 \%$ \\
\hline Total patients $(\mathrm{n}=267)$ & $44.19 \%(\mathrm{n}=118)$ & $46.82 \%(\mathrm{n}=125)$ & $8.99 \%(\mathrm{n}=24)$ & $67.60 \%$ & \\
\hline
\end{tabular}

Table 4: Allele and genotype frequencies of CYP2C19*3(636G>A).

\begin{tabular}{|c|c|c|c|c|c|}
\hline \multirow{2}{*}{} & \multicolumn{2}{|c|}{ Genotype frequencies } & \multicolumn{2}{c|}{ Allele frequencies } \\
\cline { 2 - 6 } & GG & GA & AA & G & A \\
\hline Males $(n=196)$ & $86.73 \%(n=170)$ & $12.76 \%(n=25)$ & $0.51 \%(n=1)$ & $93.11 \%$ & $3.59 \%$ \\
\hline Females $(n=71)$ & $94.37 \%(n=67)$ & $4.22 \%(n=3)$ & $1.41 \%(n=1)$ & $96.48 \%$ & $5.99 \%$ \\
\hline Total patients $(n=267)$ & $88.76 \%(n=237)$ & $10.49 \%(n=28)$ & $0.75 \%(n=2)$ & $94.01 \%$ & \\
\hline
\end{tabular}

Table 5: Allele and genotype frequencies of CYP2C19*17(-806C $>$ T).

\begin{tabular}{|c|c|c|c|c|c|}
\hline \multirow{2}{*}{} & \multicolumn{2}{|c|}{ Genotype frequencies } & \multicolumn{2}{c|}{ Allele frequencies } \\
\cline { 2 - 6 } & CC & CT & TT & C & T \\
\hline Males $(n=112)$ & $95.54 \%(n=107)$ & $4.46 \%(n=5)$ & $0 \%(n=0)$ & $97.77 \%$ & $2.23 \%$ \\
\hline Females $(n=39)$ & $94.87 \%(n=37)$ & $5.13 \%(n=2)$ & $0 \%(n=0)$ & $97.44 \%$ & $2.56 \%$ \\
\hline Total patients $(n=151)$ & $95.36 \%(n=144)$ & $4.64 \%(n=7)$ & $0 \%(n=0)$ & $97.68 \%$ & $2.32 \%$ \\
\hline
\end{tabular}

Table 6: Metabolic phenotype frequencies of CYP2C19.

\begin{tabular}{|c|c|c|c|c|c|c|c|}
\hline & \multicolumn{7}{|c|}{ Metabolic phenotype frequencies } \\
\hline & $* 1 / *_{1}$ & $* 1 /{ }^{*} 2$ & $* 1 / * 3$ & $* 2 / * 2$ & $* 2 / * 3$ & $* 3 / * 3$ & $* 1 /{ }^{*} 17$ \\
\hline Males $(n=112)$ & $36.61 \%(n=41)$ & $41.07 \%(n=46)$ & $8.04 \%(n=9)$ & $4.46 \%(n=5)$ & $6.25 \%(n=7)$ & $0.89 \%(\mathrm{n}=1)$ & $2.68 \%(n=3)$ \\
\hline Females $(n=40)$ & $30 \%(n=12)$ & $45 \%(n=18)$ & $0 \%(\mathrm{n}=0)$ & $15 \%(n=6)$ & $5 \%(n=2)$ & $2.5 \%(n=1)$ & $2.5 \%(n=1)$ \\
\hline Total patients $(n=152)$ & $34.87 \%(n=53)$ & $42.10 \%(n=64)$ & $5.92 \%(n=9)$ & $7.24 \%(n=11)$ & $5.92 \%(n=9)$ & $1.32 \%(n=2)$ & $2.63 \%(n=4)$ \\
\hline
\end{tabular}

Table 7: Allele and genotype frequencies of PON1 (A>G).

\begin{tabular}{|c|c|c|c|c|}
\hline \multirow{2}{*}{} & \multicolumn{3}{|c|}{ Genotype frequencies } & Allele frequencies \\
\cline { 2 - 5 } & AA & AG & A & $34.67 \%$ \\
\hline Males $(n=150)$ & $10 \%(n=15)$ & $49.33 \%(n=74)$ & $40.67 \%(n=61)$ & $65.33 \%$ \\
\hline Females $(n=55)$ & $16.36 \%(n=9)$ & $47.27 \%(n=26)$ & $36.37 \%(n=20)$ & $40 \%$ \\
\hline Total patients $(n=205)$ & $11.71 \%(n=24)$ & $48.78 \%(n=100)$ & $39.51 \%(n=81)$ & $36.10 \%$ \\
\hline
\end{tabular}

\section{References}

1. Sullivan Klose TH, Ghanayem BI, Bell DA, Zhang ZY, Kaminsky LS, et al. (1996) The role of the CYP2C9-Leu359 allelic variant in the tolbutamide polymorphism. Pharmacogenetics 6(4): 341.

2. Wang SL, Huang J, Lai MD, Tsai JJ (1995) Detection of CYP2C9 polymorphism based on the polymerase chain reaction in Chinese. Pharmacogenetics 5(1): 37-42.

3. Beitelshees AL, Horenstein RB, Vesely MR, Mehra MR, Shuldiner AR (2011) Pharmacogenetics and clopidogrel response in patients undergoing percutaneous coronary interventions. Clin Pharmacol Ther 89(3): 455 .

4. Sim SC, Risinger C, Dahl ML, Aklillu E, Christensen M, et al. (2006) A common novel CYP2C19 gene variant causes ultrarapid drug metabolism relevant for the drug response to proton pump inhibitors and antidepressants. Clin Pharmacol Ther 79(1): 103.

5. Ma W, Liang Y, Zhu J, Chen T, Feng G, et al. (2016) Relationship of paraoxonase-1 Q192R genotypes and in-stent restenosis and re-stenting in Chinese patients after coronary stenting. Atherosclerosis 251: 305.

Cite this article: Keguang C, Rui Z, Nan G, Rui-chen G. Genotype and Allele Frequencies of CYP2C9, VKORC1, CYP2C19 and PON1 in Chinese 


\section{ISSN: 2574-1241}

DOI: 10.26717/BJSTR.2018.12.002185

Rui-chen Guo. Biomed J Sci \& Tech Res

(C) This work is licensed under Creative

Submission Link: https://biomedres.us/submit-manuscript.php

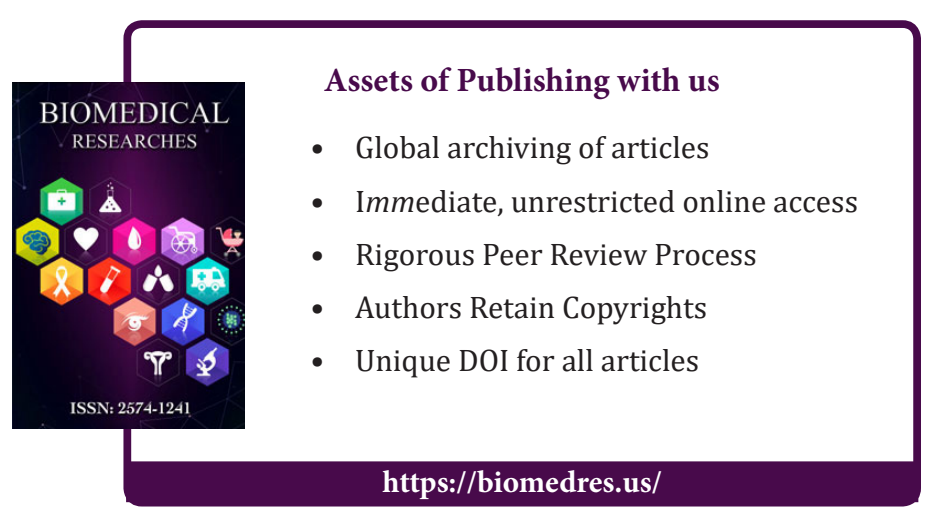

